\title{
Light-Cone Sum Rules with $B$-Meson Distribution Amplitudes
}

Alexander Khodjamirian (University of Siegen)

(with Thomas Mannel and Niels Offen)

Continuous Advances in QCD, FTPI, Minneapolis, May 11-14, 2006 


\section{QCD light-cone sum rules (LCSR)}

Balitsky, Braun, Kolesnichenko; Braun, Filyanov (1989);

Chernyak, Zhitnisky(1990)

allow to calculate hadronic form factors:

several important applications to exclusive $B$ decays

based on:

- vacuum $\rightarrow$ hadron correlators

- OPE near the light-cone,

- inputs: light-cone distribution amplitudes of $\pi, K, \rho, \ldots$

- dispersion relations $\oplus$ parton-hadron duality

"remake" of QCD sum rules: Shifman, Vainshtein, Zakharov (1979) based on vacuum $\rightarrow$ vacuum correlators, OPE in local operators, inputs: quark/gluon condensates 


\section{LCSR for $B \rightarrow \pi$ form factor}

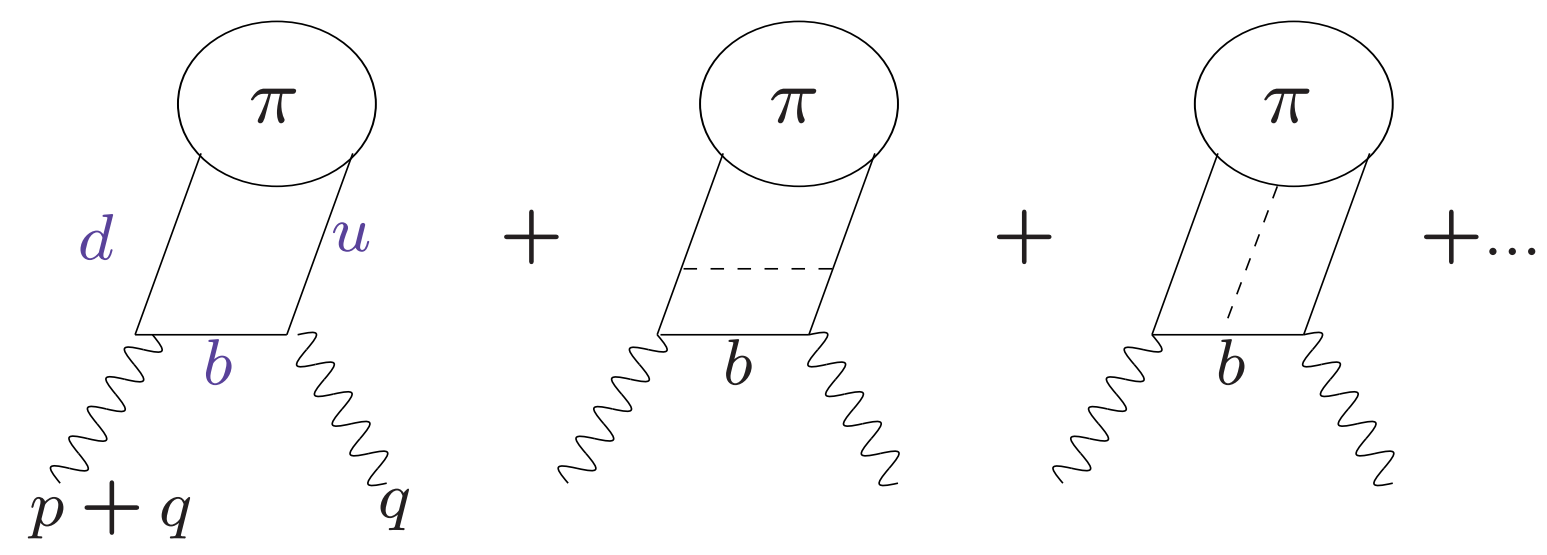

The correlation function: $\quad q^{2},(p+q)^{2} \ll m_{b}^{2}, \quad b$-quark highly virtual

$$
F_{\lambda}(q, p)=i \int d^{4} x e^{i q x}\left\langle\pi(p)\left|T\left\{\bar{u}(x) \gamma_{\lambda} b(x), \bar{b}(0) i \gamma_{5} d(0)\right\}\right| 0\right\rangle
$$

operator-product-expansion ( $\mathrm{OPE}$ ) near the light-cone, $x^{2} \sim 0$ 


\section{OPE near the light-cone}

schematically,

$$
\begin{aligned}
F^{(B)}(q, p)=i \int d^{4} x e^{i q x}\left\{\left.\left[S_{0}\left(x^{2}, m_{b}^{2}, \mu\right)+\alpha_{s} S_{1}\left(x^{2}, m_{b}^{2}, \mu\right)\right] \otimes\langle\pi(p)|\bar{u}(x) \Gamma d(0)| 0\rangle\right|_{\mu}\right. \\
\left.\left.+\int_{0}^{1} d v \tilde{S}\left(x^{2}, m_{b}^{2}, \mu, v\right) \otimes\langle\pi(p)| \bar{u}(x) G(v x) \tilde{\Gamma} d(0)\right\}\left.|0\rangle\right|_{\mu}\right\}+\ldots
\end{aligned}
$$

$* S_{0,1}, \tilde{S}$ - perturbative amplitudes, (virtual $b$-quark)

* universal distribution amplitudes of $\pi$ (or $K, \rho, K^{*}$ ):

$$
\left\langle\pi(q)\left|\bar{u}(x)[x, 0] \gamma_{\mu} \gamma_{5} d(0)\right| 0\right\rangle_{x^{2}=0}=-i q_{\mu} f_{\pi} \int_{0}^{1} d u e^{i u q x} \varphi_{\pi}(u)+O\left(x^{2}\right) .
$$

* the expansion (light-cone OPE) goes over $\alpha_{s}(\mu)$ and powers of $1 / \mu^{2}$;

* typical scale $\mu^{2} \sim m_{b} \Lambda$, where $\Lambda \sim 1 \mathrm{GeV} \gg \Lambda_{Q C D}$ 


\section{Derivation of LCSR}

- Hadronic dispersion relation in $(p+q)^{2}:\left(q^{2} \ll m_{b}^{2}\right.$ fixed $)$

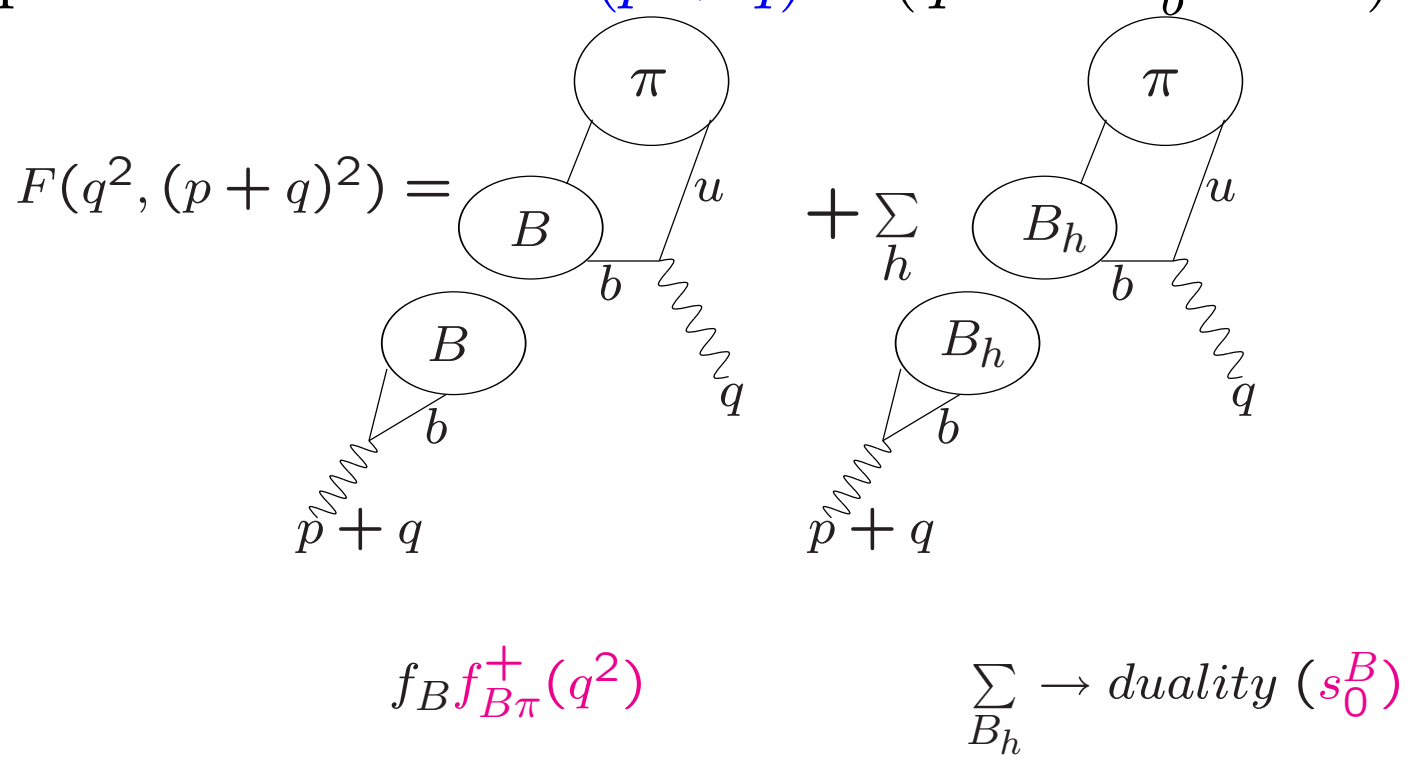

- $\left[f_{B \pi}\left(q^{2}\right)\right]_{L C S R}$ includes both "soft" (end-point) and "hard" $\left(\sim \alpha_{s}\right)$ contributions, valid at $0<q^{2}<m_{B}^{2}-\mu^{2}$

- more details/results: talk by Roman Zwicky 


\section{New approach: LCSR with $B$ meson DA}

A.K., T. Mannel, N.Offen PLB(2005), hep-ph/0504091

also (in SCET): F. De Fazio, T. Feldmann and T. Hurth; hep-ph/0504088

- The "inversed" correlator:

$B$ meson on-shell, pion interpolated with an axial current $\oplus$ duality

$$
F_{\mu \nu}^{(B)}(p, q)=i \int d^{4} x e^{i p \cdot x}\left\langle 0\left|T\left\{\bar{d}(x) \gamma_{\mu} \gamma_{5} u(x), \bar{u}(0) \gamma_{\nu} b(0)\right\}\right| \bar{B}^{0}(p+q)\right\rangle .
$$
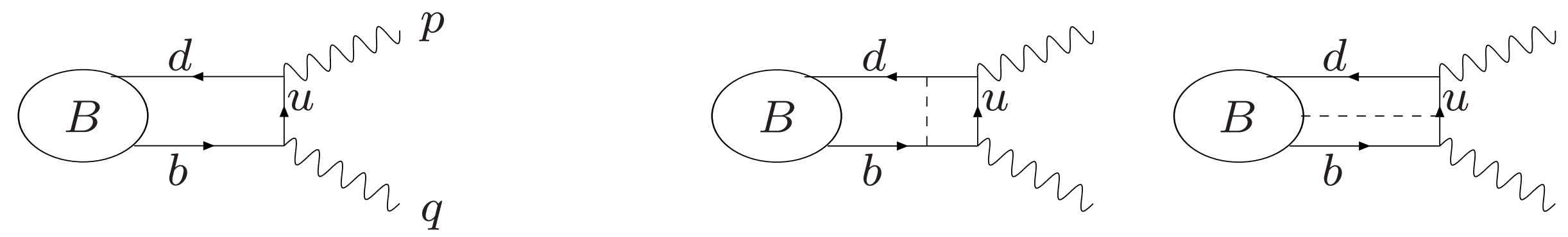

$q^{2}=0, p^{2}<0,\left|p^{2}\right| \gg \Lambda_{Q C D}^{2}, \quad u$-quark propagates near LC . 


\section{OPE near the light-cone}

schematically,

$$
\begin{array}{r}
F(q, p)=i \int d^{4} x e^{i q x}\left\{\left.\left[S_{0}\left(x^{2}, \mu\right)+\alpha_{s} S_{1}\left(x^{2}, \mu\right)\right] \otimes\langle 0|\bar{d}(x) \Gamma b(0)| B(v)\rangle\right|_{\mu}\right. \\
\left.\left.\quad+\int_{0}^{1} d v \tilde{S}\left(x^{2}, m_{b}^{2}, \mu, v\right) \otimes\langle 0| \bar{d}(x) G(v x) \tilde{\Gamma} b(0)\right\}\left.|B(v)\rangle\right|_{\mu}\right\}+\ldots
\end{array}
$$

$* S_{0,1}, \tilde{S}$ - perturbative amplitudes, ( virtual $u$-quark)

* universal distribution amplitudes of $B(v):\langle 0|\bar{d}(x)[x, 0] \Gamma b(0)| B(v)\rangle_{x^{2}=0}$

- first uses of $B$-meson DA in PQCD factorization for $B \rightarrow \pi$

A. Szczepaniak, E. M. Henley and S. J. Brodsky (1990) R. Akhoury, G. Sterman and Y. P. Yao (1994) 


\section{$B$-Meson two-particle DA: the definition}

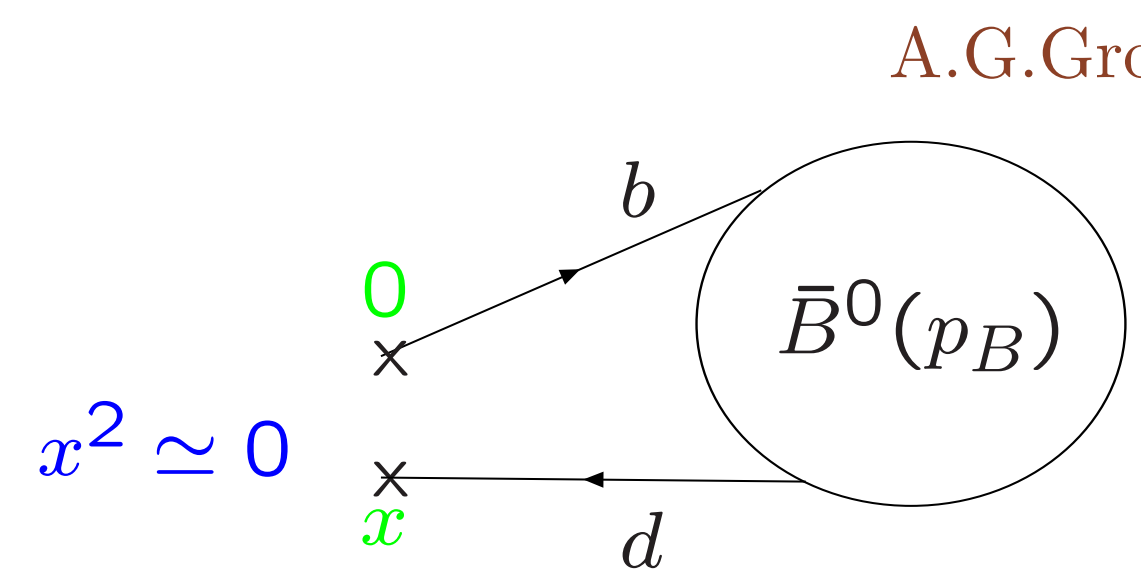

- Light-cone matrix element, consistent with HQET

$$
\begin{aligned}
& \left.\left\langle 0\left|T\left\{\bar{d}_{\alpha}(x)[x, 0] b_{\beta}(0)\right\}\right| \bar{B}^{0}(v)\right\rangle\right|_{x^{2}=0} \\
& =-\frac{i f_{B} m_{B}}{4}\left[(1+\psi) \gamma_{5} \int_{0}^{\infty} d \omega e^{-i \omega v \cdot x}\left\{\phi_{+}^{B}(\omega)+\frac{\phi_{+}^{B}(\omega)-\phi_{-}^{B}(\omega)}{2 v \cdot x} \not x\right\} \gamma_{5}\right]_{\beta \alpha},
\end{aligned}
$$

$[x, 0]$-Wilson line, two normalized DA's $\phi_{+}^{B}(\omega)$ and $\phi_{-}^{B}(\omega)$,

- variable $\omega=\left(l_{0}+l_{3}\right)$ : (l-light spectator momentum in $B$ rest frame) 


\section{Factorization in $B \rightarrow \gamma l \nu_{l}$}

$$
\left(p_{l}+p_{\nu}\right)^{2} \sim 0, E_{\gamma} \sim m_{B} / 2
$$

$$
A(B \rightarrow \gamma l \nu) \sim \int d \omega \phi_{+}^{B}(\omega) T_{h}(\omega)
$$

$T_{h} \sim 1 / \omega$

$$
1 / \lambda_{B}=\int_{0}^{\infty} d \omega \frac{\phi_{+}^{B}(\omega)}{\omega}
$$

- the inverse moment

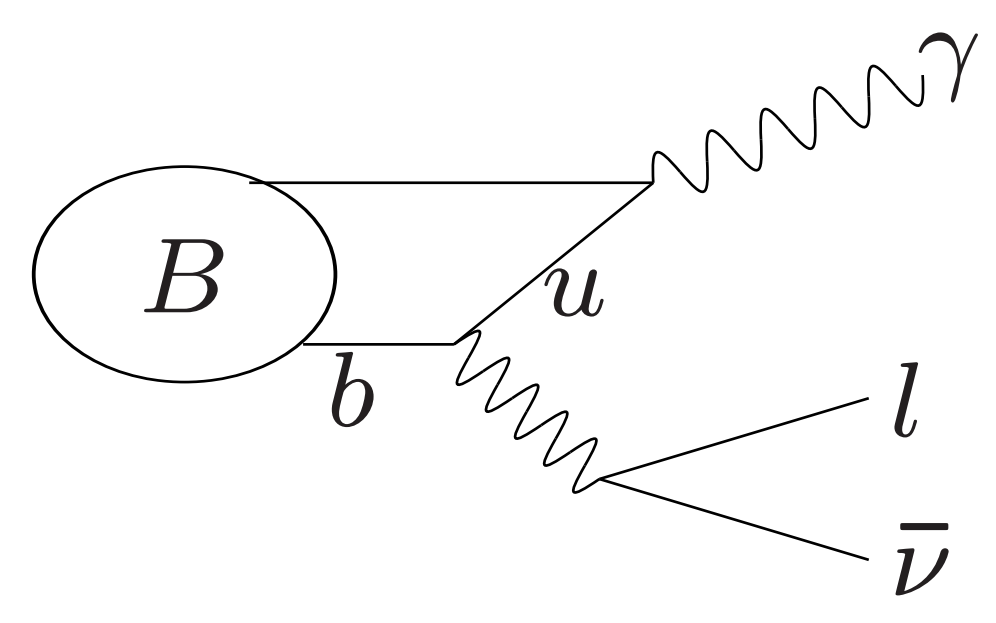

G. P. Korchemsky, D. Pirjol, T. M. Yan (2000)

S. Descotes-Genon, C. T. Sachrajda (2003)]

S. W. Bosch, R. J. Hill, B. O. Lange, M. Neubert (2004)

- factorization in $B \rightarrow \pi, B \rightarrow h_{1} h_{2}$ etc. 


\section{Quark-antiquark-gluon DA's: definition}

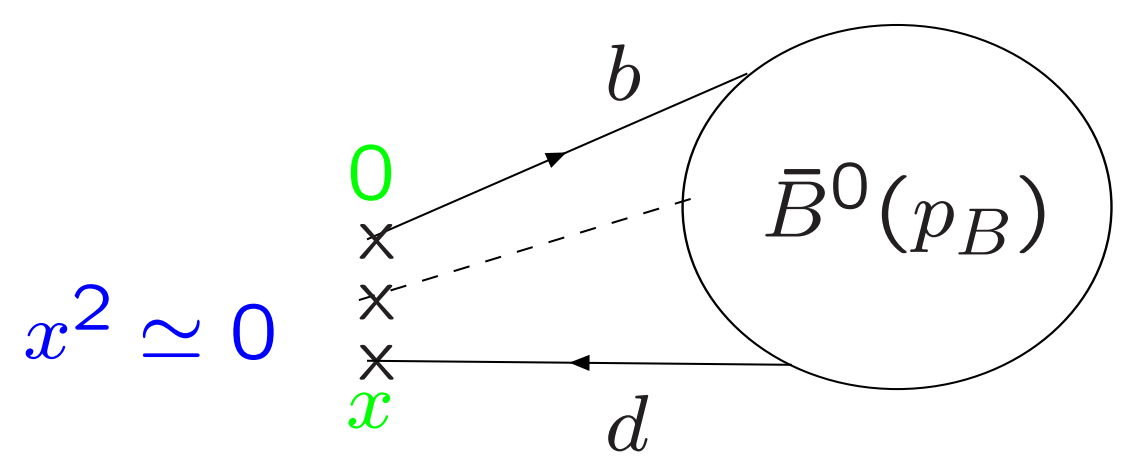

H. Kawamura, J. Kodaira, C.F.Qiao and K. Tanaka,(2001)

$$
\begin{aligned}
& \left\langle 0\left|\bar{d}_{\alpha}(x) G_{\lambda \rho}(u x) b_{\beta}(0)\right| \bar{B}^{0}(v)\right\rangle=\frac{f_{B} m_{B}}{4} \int_{0}^{\infty} d \omega \int_{0}^{\infty} d \xi e^{-i(\omega+u \xi) v \cdot x} \\
& \times\left[( 1 + \psi ) \left\{\left(v_{\lambda} \gamma_{\rho}-v_{\rho} \gamma_{\lambda}\right)\left(\Psi_{A}(\omega, \xi)-\Psi_{V}(\omega, \xi)\right)-i \sigma_{\lambda \rho} \Psi_{V}(\omega, \xi)\right.\right. \\
& \left.\left.-\left(\frac{x_{\lambda} v_{\rho}-x_{\rho} v_{\lambda}}{v \cdot x}\right) X_{A}(\omega, \xi)+\left(\frac{x_{\lambda} \gamma_{\rho}-x_{\rho} \gamma_{\lambda}}{v \cdot x}\right) Y_{A}(\omega, \xi)\right\}\right]_{\beta \alpha} .
\end{aligned}
$$




\section{What do we know about $B$-meson DA's}

- model-independent relations from QCD equation of motion, e.g. Wandzura-Wilczek-type:

$$
\phi_{-}^{B}(\omega)=\int_{\omega}^{\infty} d \rho \frac{\phi_{+}^{B}(\rho)}{\rho}+\int d \omega d \xi\left\{\Psi_{V, A}(\omega, \xi)\right\} \Rightarrow \phi_{-}^{B}(0)=1 / \lambda_{B}+\{\text { corr. }\}
$$

- boundary conditions: $\omega \rightarrow 0$ : $\phi_{+}^{B}(\omega) \sim \omega, \quad \phi_{-}^{B}(0)=$ const

- Evolution of $\phi_{+}^{B}(\omega, \mu)$ calculated in HQET is nontrivial, $\Rightarrow \lim _{\omega \rightarrow \infty} \phi(\omega) \sim-\log (\omega / \mu) / \omega$, "radiative tail" [M. Neubert, B. Lange, (2003)]

- no parton interpretation, positive moments divergent, but $\lambda_{B}(\mu)$ well defined in $O\left(\alpha_{s}\right)$

- no problem for the new sum rules containing integrals over small $\omega<s_{0} / m_{B}$ 
- models of $\phi_{ \pm}^{B}(\omega)$ based on QCD sum rules in HQET

[ A. G. Grozin and M. Neubert (1997)]

- The correlator for $\phi_{+}^{B}(\omega)$ :

$$
\begin{aligned}
& \quad i \int d^{4} x e^{-i k(v x)}\left\langle 0\left|\mathrm{~T}\left\{O_{+}(t) \bar{h}_{v}(x) \Gamma_{2} q(x)\right\}\right| 0\right\rangle=\{\ldots\} T(t, k) . \\
& O_{+}(t)=\bar{q}(t n) \not \not[t n, 0] \Gamma h_{v}(0), \\
& k<0 \text { - external (Euclidean) momentum variable, } \\
& k=\bar{\Lambda} \text { is } B \text { meson pole in HQET },\{\ldots\} \text { - a trace }
\end{aligned}
$$

- loop $\oplus$ condensate $\Rightarrow$ simple ansatz

$$
\phi_{+}^{B}(\omega)=\left(\omega / \omega_{0}^{2}\right) e^{\left(-\omega / \omega_{0}\right)}, \quad \phi_{-}^{B}(\omega)=\left(1 / \omega_{0}\right) e^{\left(-\omega / \omega_{0}\right)},
$$

- A hybrid model for $\phi(\omega, \mu)$ (exponent.ansatz with the radiative tail) S.J. Lee, M. Neubert, (2005) 
- NLO calculation (including radiative corrections)

[ V. M. Braun, D. Y. Ivanov and G. P. Korchemsky,(2003)]

- the sum rule fitted to an explicit ansatz for $\varphi_{+}^{B}(\omega)$,

$$
\phi_{+}^{B}(\omega, \mu=1 \mathrm{GeV})=\frac{4 \lambda_{B}^{-1}}{\pi} \frac{\omega}{\omega^{2}+1}\left[\frac{1}{\omega^{2}+1}-\frac{2\left(\sigma_{B}-1\right)}{\pi^{2}} \ln \omega\right],
$$

$(\omega$ in units of $\mathrm{GeV}) \lambda_{B}=(460 \pm 110) \mathrm{MeV}, \sigma_{B}=1.4 \pm 0.4$ at $\mu=1 \mathrm{GeV}$

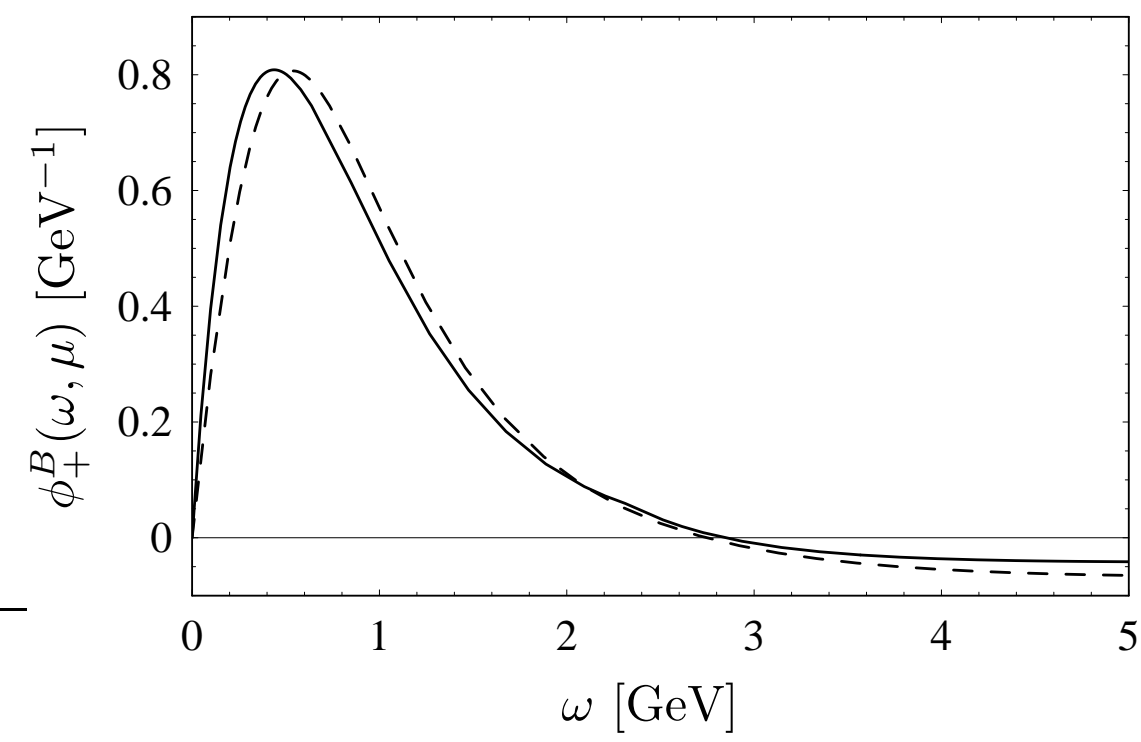

solid (dashed) is the Lee-Neubert (Braun-Ivanov-Korchemsky) 


\section{Deriving the simplest sum rule}

- OPE result for $B \rightarrow \pi$, the LO diagram: only $\phi_{-}^{B}(\omega)$ contributes

$$
F_{\mu \nu}^{(B)}=2 i f_{B} \int_{0}^{\infty} \frac{d \omega}{m_{B} \omega-p^{2}} \phi_{-}^{B}(\omega) p_{\mu} p_{\nu}+\ldots
$$

- Hadronic dispersion relation:

$$
\begin{aligned}
F_{\mu \nu}^{(B)} & =\left\langle 0\left|\bar{d} \gamma_{\mu} u\right| \pi(p)\right\rangle\left\langle\pi(p)\left|\bar{u} \gamma_{\nu} b\right| B(p+q)\right\rangle+\ldots \\
& =\left\{\frac{2 i f_{\pi} f_{B \pi}^{+}(0)}{-p^{2}}+\int_{s_{h}}^{\infty} d s \frac{\rho^{h}(s)}{s-p^{2}}\right\} p_{\mu} p_{\nu}+\ldots,
\end{aligned}
$$

apply duality in pion channel $\oplus$ Borel transformation. 
- The relation between $B$ meson parameters: (using $s_{\pi}^{0} \ll m_{B}^{2}$ ):

$$
\frac{1}{\lambda_{B}} \simeq \frac{f_{\pi} f_{B \pi}^{+}(0) m_{B}}{f_{B} M^{2}\left(1-e^{s_{0}^{\pi} / M^{2}}\right)} .
$$

- inputs: use LCSR for $B \rightarrow \pi$ form factor (in terms of pion DA's), 2pt sum rule for $f_{B}$ and predict $\lambda_{B}$

- 3-particle $B$ meson DA's, enter

1) soft-gluon diagram

2) indirectly, violation of WW relation estimated - a few $\%$ 


\section{Summary on the inverse moment}

$1 / \lambda_{B}=\int_{0}^{\infty} d \omega \frac{\phi_{+}^{B}(\omega)}{\omega}$ renorm. scale $\sim 1 \mathrm{GeV}$

\begin{tabular}{l|c|c|}
\hline \hline Method & $\lambda_{B}[\mathrm{MeV}]$ & Ref. \\
\hline 2pt SR in HQET,LO & $\simeq 350$ & Grozin,Neubert \\
\hline 2pt SR in HQET, NLO & $440 \pm 110$ & Braun, Ivanov,Korchemsky \\
\hline LCSR for $B \rightarrow \gamma l \nu_{l}$ & $\simeq 600$ & Ball, Kou \\
\hline "inverted" LCSR for $B \rightarrow \pi$ & $460 \pm 160$ & A.K.,Mannel, Offen \\
\hline first moments + Ansatz & $480 \pm 55$ & Lee, Neubert \\
\hline
\end{tabular}


- the new method allows to calculate many different $B \rightarrow$ light form factors "in one go", including SU(3) breaking, $m_{q}=m_{s}$

- the main advantage: knowledge of pion, $K, \rho, K^{*}$ DA's not needed, decay constants, duality thresholds from exp. and/or two-point (SVZ) SR

- contributions of 3-particle DA's of $B$ meson suppressed by powers of $\sqrt{s_{0}} / m_{B}$ (work in progress),

- preliminary (sample) results for all major heavy-light form factors:

inputs: 2-particle DA,s $\phi_{ \pm}^{B}(\omega)$, Grozin-Neubert exponential ansatz, $\lambda_{B}=440 \mathrm{MeV}, f_{B}=180 \mathrm{MeV}, M^{2}=1 \mathrm{GeV}, m_{s}(1 \mathrm{GeV})=130 \mathrm{MeV}$ more detailed analysis in progress

- compared with the results of conventional LCSR [BZ] by P. Ball, R. Zwicky, (2005) $\left(B \rightarrow \pi, K, \rho, K^{*}\right)$ 


\begin{tabular}{|c|c|c|c|}
\hline Form Factor & LCSR & LCSR & Ref. \\
& with $B \mathrm{DA}^{\prime}($ prelim) & with light-meson DA's & \\
\hline$f_{B \pi}^{+}(0)$ & $0.267 \pm$ & $0.258 \pm 0.03$ & {$[\mathrm{BZ}]$} \\
& & $0.26 \pm 0.05$ & {$[\mathrm{KMMM}]$} \\
& & $0.25 \pm 0.05$ & {$[\mathrm{AGRS}]$} \\
\hline$f_{B K}^{+}(0)$ & $0.328 \pm$ & $0.301 \pm 0.041$ & {$[\mathrm{BZ}]$} \\
\hline$f_{B \pi}^{T}(0)$ & $0.24 \pm$ & $0.253 \pm 0.028$ & {$[\mathrm{BZ}]$} \\
\hline$f_{B K}^{T}(0)$ & $0.305 \pm$ & $0.328 \pm 0.04$ & {$[\mathrm{BZ}]$} \\
\hline$V^{B \rho}(0)$ & $0.382 \pm$ & $0.323 \pm 0.029$ & {$[\mathrm{BZ}]$} \\
\hline$V^{B K^{*}}(0)$ & $0.442 \pm$ & $0.411 \pm 0.033$ & {$[\mathrm{BZ}]$} \\
\hline$A_{1}^{B \rho}(0)$ & $0.281 \pm$ & $0.242 \pm 0.024$ & {$[\mathrm{BZ}]$} \\
\hline$A_{1}^{B K^{*}}(0)$ & $0.328 \pm$ & $0.292 \pm 0.028$ & {$[\mathrm{BZ}]$} \\
\hline$A_{2}^{B \rho}(0)$ & $0.253 \pm$ & $0.221 \pm 0.023$ & {$[\mathrm{BZ}]$} \\
\hline$A_{2}^{B K^{*}}(0)$ & $0.304 \pm$ & $0.259 \pm 0.027$ & {$[\mathrm{BZ}]$} \\
\hline$T_{1}^{B \rho}(0)$ & $0.323 \pm$ & $0.267 \pm 0.021$ & {$[\mathrm{BZ}]$} \\
\hline$T_{1}^{B K^{*}}(0)$ & $0.375 \pm$ & $0.333 \pm 0.028$ & {$[\mathrm{BZ}]$} \\
\hline
\end{tabular}

*[KMMM] A. K.,T. Mannel, M. Melcher and B. Melic, PRD (2005), hep-ph/0509049

*[AGRS] Arnesen, Grinstein, Rothstein, Stuart, hep-ph/0504209 


\section{Conclusions}

- new type of LCSR, calculating $B \rightarrow$ light form factors:

already leading order has a good agreement with LCSR with light meson DA's

- quantitative estimates of SU(3) breaking effects;

- sensitivity to the inverse moment $\lambda_{B}$, values $<300 M e V$ disfavored 